\title{
EXPANSION PROBLEMS ASSOCIATED WITH A SYSTEM OF INTEGRAL EQUATIONS*
}

\author{
BY \\ WILLIAM T. REID $\dagger$
}

1. Introduction. Fredholm $\ddagger$ has shown how a system of linear integral equations of the form

$$
y^{(i)}(x)=\lambda \int_{a}^{b} \sum_{\alpha=1}^{n} K_{i \alpha}(x ; s) y^{(\alpha)}(s) d s+f^{(i)}(x) \quad(i=1,2, \cdots, n)
$$

may be reduced to a single linear integral equation whose kernel is defined on $a \leqq x \leqq a+n(b-a), a \leqq s \leqq a+n(b-a)$. Greggi§ considered a system of the form (1) and by use of the transformation introduced by Fredholm showed the form of the resolvent matrix for the system; for the symmetric system where $K_{i j}(x ; s)=K_{j i}(s ; x)(i, j=1,2, \cdots, n)$ he also stated theorems analogous to those proved by Schmidt $\|$ for a single integral equation with symmetric kernel. System (1) also comes under the class of systems treated by Platrier. $\uparrow$

Weatherburn** has treated the system (1) without using the transformation introduced by Fredholm, but by vector method throughout. He states all results for the case $n=3$, but his method of procedure is equally applicable to the general case.

In the present paper a special system of the form (1), to which is applied the term "definitely self-adjoint," is considered and the existence of a countable infinity of real characteristic numbers is established, together with expansion theorems in terms of the characteristic solutions of the system of integral equations. A definitely self-adjoint system of integral equations includes as a special case the symmetric system with closed matrix kernel. It also includes the system of integral equations to which a boundary value problem for a system of ordinary linear differential equations of the first order which

* Presented to the Society, April 18, 1930; received by the editors July 21, 1930.

† National Research Fellow in Mathematics.

‡ I. Fredholm, Acta Mathematica, vol. 27 (1903), pp. 365-390. 551.

\$ G. Greggi, Atti del Reale Istituto Veneto di Scienze, Lettere ed Arti, vol. 71 (1912), pp. 541-

II E. Schmidt, Dissertation, Göttingen, 1905.

If Ch. Platrier, Journal de Mathématiques, (6), vol. 9 (1913), pp. 233-304; in particular, pp. 257-266.

** C. E. Weatherburn, Transactions of the Cambridge Philosophical Society, vol. 22 (19121923), pp. 133-158. 
is definitely self-adjoint in the sense defined by Bliss* may be reduced by the introduction of the Green's matrix of the differential system. It is also shown that the idea of definite self-adjointness as defined by Bliss may be extended to a somewhat more general differential system, and that the system of integral equations to which the more general boundary value problem may be reduced by the introduction of the Green's matrix is also of the definitely selfadjoint type considered in this paper.

Matrix notation is used throughout this paper. All matrices used are denoted by capital letters and are supposed to have $n$ rows and $n$ columns, the element in the $i$ th row and the $j$ th column being denoted by the same letter with the subscript $i j$. Likewise, all vectors are supposed to have $n$ components, and if $v$ is a vector then $v^{(i)}$ is used to denote the $i$ th component of $v$. If $M$ is a matrix and $v$ is a vector, then $M v$ denotes the vector whose $i$ th component is $M_{i \alpha} v^{(\alpha)}$, where it is understood that $\alpha$ is an umbral label, i.e., its repetition in an expression indicates summation with respect to that label over the values $1,2, \cdots, n$. Similarly, $v M$ denotes the vector whose $i$ th component is $v^{(\alpha)} M_{\alpha i}$. If $M$ and $N$ are matrices, then $M+N$ is the matrix $\left\|M_{i j}+N_{i j}\right\|$ and $M N$ denotes the product matrix $\left\|M_{i \alpha} N_{\alpha j}\right\|$; also, if $u$ and $v$ are vectors, then $u v$ denotes the scalar quantity $u^{(\alpha)} v^{(\alpha)}$.

2. The Fredholm determinant and resolvent matrix. We may write system (1) in vector form as

$$
y(x)=\lambda \int_{a}^{b} K(x ; s) y(s) d s+f(x),
$$

where $y(x) \equiv\left(y^{(\alpha)}(x)\right)$ and $f(x) \equiv\left(f^{(\alpha)}(x)\right)$ are vectors each of whose components are continuous on $X: a \leqq x \leqq b$, and $K(x ; s) \equiv\left\|K_{i j}(x ; s)\right\|$ is a matrix. We will suppose that the discontinuities of each element $K_{i j}(x ; s)(i, j=1$, $2, \cdots, n)$ are regularly distributed $\dagger$ in the square $W: a \leqq x \leqq b, a \leqq s \leqq b$, and that $\left|K_{i j}(x ; s)\right|$ is bounded by a finite constant on $W$; finally, that $K_{i i}(x ; x)$ is Riemann integrable on $X$.

As for a single integral equation we may prove for the vector equation (2) the existence of the Fredholm determinant $D(\lambda)$, which is a permanently converging series in $\lambda$, and the existence of the resolvent matrix $D(x ; s \mid \lambda)$ $\equiv\left\|D_{i j}(x ; s \mid \lambda)\right\|$, each element of which converges absolutely and uniformly in $W$ for all values of $\lambda$.

* G. A. Bliss, these Transactions, vol. 28 (1926), pp. 561-584.

$\dagger$ The discontinuities of a function of $(x, s)$ are said to be regularly distributed in $W$ if they all lie on 2 finite number of curves which have continuous tangents and no one of which is met by a line parallel to the axis of $x$ or to the axis of $s$ in more than a finite number of points. See Bôcher, $A n$ Introduction to the Study of Integral Equations, London, 1909, p. 3. 
The matrix relations

$$
\begin{aligned}
D(x ; s \mid \lambda)-D(\lambda) K(x ; s) & =\lambda \int_{a}^{b} K(x ; t) D(t ; s \mid \lambda) d t, \\
& =\lambda \int_{a}^{b} D(x ; t \mid \lambda) K(t ; s) d t,
\end{aligned}
$$

and the scalar relation

$$
\frac{d^{p}}{d \lambda^{p}} D(\lambda)=-\int_{a}^{b} \frac{\partial^{p-1}}{\partial \lambda^{p-1}}\left[\sum_{\alpha=1}^{n} D_{\alpha \alpha}(t ; t \mid \lambda)\right] d t \quad(p=1,2, \cdots)
$$

may be established by the same method that is used to prove the analogous relations for the single integral equation. The following theorem may also be proved, as for a single integral equation.*

THEOREM A. If $\lambda=\lambda_{0}$ is not a root of $D(\lambda)=0$, then (2) has a unique solution $y\left(x, \lambda_{0}\right)$ for $\lambda=\lambda_{0}$, and

$$
y\left(x, \lambda_{0}\right)=f(x)+\lambda_{0} \int_{a}^{b} \frac{D\left(x ; s \mid \lambda_{0}\right)}{D\left(\lambda_{0}\right)} f(s) d s .
$$

If $\lambda_{0}$ is a root of $D(\lambda)=0$ of multiplicity $m$, then the equation

$$
y(x)=\lambda \int_{a}^{b} K(x ; s) y(s) d s
$$

has $k(0<k \leqq m)$ linearly independent solutions for $\lambda=\lambda_{0}$; furthermore, the associated vector integral equation

$$
z(x)=\lambda \int_{a}^{b} z(s) K(s ; x) d s
$$

also has $k$ linearly independent solutions for $\lambda=\lambda_{0}$. If $D\left(\lambda_{0}\right)=0$, then a necessary and sufficient condition that (2) have a solution for $\lambda=\lambda_{0}$ is that for every solution $z(x)$ of $(8)$ for $\lambda=\lambda_{0}$, we have

$$
\int_{a}^{b} z(x) f(x) d x=0 .
$$

3. The definitely self-adjoint vector integral equation. Throughout this section $f(x)$ and $g(x)$ are used to denote arbitrarily selected vectors whose components are continuous on $X$. Equation (7) is said to be definitely selfadjoint when the following conditions are satisfied:

* See Weatherburn, loc. cit., pp. 139-152. For a discussion of the Fredholm determinant and resolvent for a single integral equation, together with the proof of the Fredholm theorems, see Fredholm, loc. cit., Bôcher, loc. cit., pp. 38-46, or Goursat, Cours d'Analyse, Paris, vol. 3, pp. 368-380. 
(H1) $K(x ; s) \equiv H(x ; s) S(s)$, where $S(x)$ is a symmetric matrix each of whose elements is real and continuous on $X$, and the discontinuities of the real elements $H_{i j}(x ; s)$ are regularly distributed in the square $W,\left|H_{i j}(x ; s)\right|$ is bounded by a finite constant on $W$, and $H_{i i}(x ; x)$ is Riemann integrable on $X$;

(H2) $S(x) K(x ; s)=K^{*}(s ; x) S(s)$, where $K^{*}(x ; s)$ is the transposed matrix of $K(x ; s)$, i.e., $K_{i j}^{*}(x ; s)=K_{j i}(x ; s)$;

(H3) the bilinear form $\bar{v} S(x) v$, formed by the vectors $v \equiv\left(v^{(\alpha)}\right)$ and $\bar{v} \equiv\left(\bar{v}^{(\alpha)}\right)$, where $\bar{v}^{(\alpha)}$ is the conjugate imaginary of $v^{(\alpha)}(\alpha=1,2, \cdots, n)$, is non-negative on $X$ and vanishes identically for a vector $f(x)$ of the form

only when $f(x) \equiv 0$;

$$
f(x)=\int_{a}^{b} K(x ; s) g(s) d s
$$

(H4) if $\int_{a}^{b} K(x ; s) f(s) d s \equiv 0$, then $S(x) f(x) \equiv 0$ on $X$.

Now consider an equation (7) which is definitely self-adjoint according to the above definition. If $y(x)$ is a solution of (7) for a characteristic number $\lambda$, we have in view of (H2) that $y(x) S(x)$ is a solution of the associated equation (8). Therefore, if $y(x)$ and $y_{0}(x)$ are solutions of (7) corresponding to characteristic values $\lambda$ and $\lambda_{0}$, then

$$
\left[\lambda-\lambda_{0}\right] \int_{a}^{b} y_{0}(x) S(x) y(x) d x=0 .
$$

It then follows in view of (H3) that there exist no imaginary characteristic numbers for (7), and therefore

THEOREM 1. For a definitely self-adjoint vector integral equation (7) all the zeros of the Fredholm determinant $D(\lambda)$ are real and the linearly independent characteristic solutions corresponding to each zero may be chosen real.

Also, in view of (H3), we have the following theorem.

THEOREм 2. If $y_{1}(x), \cdots, y_{k}(x)$ are linearly independent solutions of a definitely self-adjoint vector integral equation (7) for a characteristic number $\lambda$, then $y_{1}(x) S(x), \cdots, y_{k}(x) S(x)$ are linearly independent solutions of (8) for the same characteristic number.

THEOREM 3. If $\lambda_{0}$ is a root of $D(\lambda)=0$ and $f(x)$ is such that

$$
y(x)=\lambda_{0} \int_{a}^{b} K(x ; s) y(s) d s+f(x)
$$

has a solution, then there is a solution of the vector equation 


$$
y(x)=\lambda \int_{a}^{b} K(x ; s) y(s) d s+f(x)
$$

which is analytic in $\lambda$ at $\lambda=\lambda_{0}$.

For since the zeros of $D(\lambda)$ are isolated, there is a neighborhood of $\lambda=\lambda_{0}$ in which there is no characteristic number of $(7)$ distinct from $\lambda_{0}$. For $\lambda$ in this neighborhood and $\lambda \neq \lambda_{0}$, the unique solution of (12) is given by

$$
y(x, \lambda)=f(x)+\lambda \int_{a}^{b} \frac{D(x ; s \mid \lambda)}{D(\lambda)} f(s) d s .
$$

We will now show that near $\lambda_{0}$ the vector $y(x, \lambda)$ is still well-defined and analytic in $\lambda$. Let $m$ be the multiplicity of $\lambda_{0}$ as a zero of $D(\lambda)$. Then $D\left(\lambda_{0}\right)=0$ $=d^{p} D\left(\lambda_{0}\right) / d \lambda^{p}(p=1,2, \cdots, m-1), d^{m} D\left(\lambda_{0}\right) / d \lambda^{m} \neq 0$, and therefore from (5) it follows that $\partial^{m-1} D\left(x ; s \mid \lambda_{0}\right) / \partial \lambda^{m-1} \not \equiv 0$ on $W$. Suppose $\partial^{r} D\left(x ; s \mid \lambda_{0}\right) / \partial \lambda^{r}$ is the first partial derivative of $D(x ; s \mid \lambda)$ which is not identically zero on $W$ for $\lambda=\lambda_{0}$. By (3), we have

$$
\frac{\partial^{r}}{\partial \lambda^{r}} D\left(x ; s \mid \lambda_{0}\right)=\lambda_{0} \int_{a}^{b} K(x ; t) \frac{\partial^{r}}{\partial \lambda^{r}} D\left(t ; s \mid \lambda_{0}\right) d t .
$$

If $r<m-1$, it would then follow that

$$
\begin{aligned}
\frac{\partial^{r+1}}{\partial \lambda^{r+1}} D\left(x ; s \mid \lambda_{0}\right)= & \lambda_{0} \int_{a}^{b} K(x ; t) \frac{\partial^{r+1}}{\partial \lambda^{r+1}} D\left(t ; s \mid \lambda_{0}\right) d t \\
& +\int_{a}^{b} K(x ; t) \frac{\partial^{r}}{\partial \lambda^{r}} D\left(t ; s \mid \lambda_{0}\right) d t \\
= & \lambda_{0} \int_{a}^{b} K(x ; t) \frac{\partial^{r+1}}{\partial \lambda^{r+1}} D\left(t ; s \mid \lambda_{0}\right) d t \\
& +\left(1 / \lambda_{0}\right) \frac{\partial^{r}}{\partial \lambda^{r}} D\left(x ; s \mid \lambda_{0}\right) .
\end{aligned}
$$

As each column of $\partial^{r} D\left(x ; s \mid \lambda_{0}\right) / \partial \lambda^{r}$ is a solution of $(7)$ for $\lambda=\lambda_{0}$, then each row of $\left[\partial^{r} D^{*}\left(x ; s \mid \lambda_{0}\right) / \partial \lambda^{r}\right] S(x)$ is a solution of (8) for $\lambda=\lambda_{0}$, and therefore, since (15) has a solution, we have from Theorem $A$ that

$$
\int_{a}^{b} \frac{\partial^{r}}{\partial \lambda^{r}} D^{*}\left(x ; s \mid \lambda_{0}\right) S(x) \frac{\partial^{r}}{\partial \lambda^{r}} D\left(x ; s \mid \lambda_{0}\right) d x=0,
$$

and, in view of (H3), that $\partial^{r} D\left(x ; s \mid \lambda_{0}\right) / \partial \lambda^{r} \equiv 0$ on $X$, which is a contradiction. Hence $r=m-1$. In view of (4) it follows that for each fixed $x$ on $X$ the rows 
of $\partial^{m-1} D\left(x ; s \mid \lambda_{0}\right) / \partial \lambda^{m-1}$ are solutions of (8) for $\lambda=\lambda_{0}$. Since, by hypothesis, there exists a solution of (11), from Theorem $\mathrm{A}$ we have

$$
\int_{a}^{b} \frac{\partial^{m-1}}{\partial \lambda^{m-1}} D\left(x ; s \mid \lambda_{0}\right) f(s) d s \equiv 0 .
$$

Hence (13) is well-defined near $\lambda_{0}$ and there exists a solution of (12) which is analytic in $\lambda$ at $\lambda=\lambda_{0}$.

THEOREM 4. If $f(x)$ is a vector which satisfies the relation

$$
\int_{a}^{b} y(x) S(x) f(x) d x=0
$$

with every characteristic solution $y(x)$ of the definitely self-adjoint equation (7), then $S(x) f(x) \equiv 0$ on $X$.

Since if $y(x)$ is a characteristic solution of (7) corresponding to a characteristic number $\lambda$, then $y(x) S(x)$ is a characteristic solution of (8) for the same characteristic number, we have in view of Theorem 2 that the relation (16) implies

$$
\int_{a}^{b} z(x) f(x) d x=0
$$

and therefore, since $\lambda=0$ is not a zero of $D(\lambda)$, that

$$
\int_{a}^{b} z(s)\left[\int_{a}^{b} K(s ; x) f(x) d x\right] d s=0
$$

for every characteristic solution $z(x)$ of (8). Then the equation

$$
y(x)=\lambda \int_{a}^{b} K(x ; s) y(s) d s+\int_{a}^{b} K(x ; s) f(s) d s
$$

has a solution for every value of $\lambda$. By Theorem 3 the solution $y(x, \lambda)$ of (17) is representable by a permanently convergent power series

$$
y(x, \lambda)=u_{0}(x)+u_{1}(x) \lambda+u_{2}(x) \lambda^{2}+\cdots .
$$

By substituting (18) in (17) and comparing coefficients, we have

$$
u_{r}(x)=\int_{a}^{b} K(x ; s) u_{r-1}(s) d s \quad(r=0,1,2, \cdots),
$$

where $u_{-1}$ is defined as $f(x)$. Let

$$
W_{r}=\int_{a}^{b} u_{0}(x) S(x) u_{r}(x) d x \quad(r=0,1,2, \cdots) .
$$


In the manner used by Bliss $\dagger$ to prove the analogous theorem for definitely self-adjoint boundary value problems, one may show that $W_{0}=0$, and therefore we have in view of (H3) that $u_{0}(x) \equiv 0$. It then follows from (H4) that $S(x) f(x) \equiv 0$ on $X$.

COROLLARY 1. If $|S(x)| \neq 0$, then $f(x) \equiv 0$ is the only continuous vector satisfying (16) with all the characteristic solutions of (7).

COROLLARY 2. The vector $f(x) \equiv 0$ is the only vector of the form

$$
f(x)=\int_{a}^{b} K(x ; s) g(s) d s
$$

which satisfies (16) with all the characteristic solutions of (7).

THEOREM 5. The totality of characteristic numbers and characteristic solutions of the definitely self-adjoint equation (7) is denumerably infinite and may be represented by $\lambda_{i}, y_{i}(x)(i=1,2, \cdots)$. Furthermore, these characteristic solutions may be chosen normed and orthogonal in the sense that

$$
\int_{a}^{b} y_{i}(x) S(x) y_{j}(x) d x=E_{i j} \quad\left(E_{i j}=0 \text { if } i \neq j, E_{i i}=1\right) .
$$

For suppose there were only a finite number, $m-1$, of characteristic solutions of (7). Denote these by $y_{1}(x), \cdots, y_{m-1}(x)$. Then $m$ continuous vectors $g_{1}(x), \cdots, g_{m}(x)$ may be chosen so that $S(x) g_{1}(x), \cdots, S(x) g_{m}(x)$ are vectors which are linearly independent on $X$. Let $f_{i}(x)=\int_{a}^{b} K(x ; s) g_{i}(s) d s(i=1$, $2, \cdots, m)$. There would then exist constants $c_{i}$ not all zero and such that $\sum_{\alpha=1}^{m} f_{\alpha}(x) c_{\alpha}$ satisfies the relation (16) with all the solutions of (7). By the above Corollary 2 it then follows that $\int_{a}^{b} K(x ; s)\left[\sum_{\alpha=1}^{m} g_{\alpha}(s) c_{\alpha}\right] d s \equiv 0$. But this, by (H2), implies that $\sum_{\alpha=1}^{m} S(x) g_{\alpha}(x) c_{\alpha} \equiv 0$, which is impossible unless $c_{i}=0(i=1,2, \cdots, m)$. Hence there is an infinity of characteristic numbers, and the number is denumerable since the zeros of $D(\lambda)$ are denumerable. The characteristic solutions of (7) may then be chosen to satisfy (21). $\neq$

For a set of characteristic solutions $y_{i}(x)$ of $(7)$ which are chosen to satisfy the relations (21), we have, in view of (H1) and (H4), the following lemmas which we state without proof.

LEMma 1. If $g(x)$ is a vector each of whose components is continuous on $X$, then the series $\sum_{\alpha=1}^{\infty}\left[\int_{a}^{b} g(x) S(x) y_{\alpha}(x) d x\right]^{2}$ converges and is not greater in value than $\int_{b}^{a} g(x) S(x) g(x) d x$.

\footnotetext{
$\dagger$ Bliss, loc. cit., pp. 573-574.

‡ See Bliss, loc. cit., p. 575; also Schmidt, loc. cit., p. 4.

$\S$ See Bliss, loc. cit., pp. 582, 583.
} 
LEMMA 2. If $h(x ; s)$ is a vector each of whose components is bounded on $W$ and for each fixed $x$ on $X$ is integrable in s on $a \leqq s \leqq b$, and $g(x)$ is a vector whose components are continuous on $X$, then the series $\sum_{\alpha=1}^{\infty}\left[\int_{a}^{b} g(s) S(s) y_{\alpha}(s) d s\right]$ $\cdot\left[\int_{a}^{b} h(x ; s) S(s) y_{\alpha}(s) d s\right]$ converges uniformly on $X$.

THEOREM 6. If $f(x)$ is a vector of the form

$$
f(x)=\int_{a}^{b} K(x ; s) g(s) d s
$$

where $g(x)$ is a vector whose components are.continuous on $X$, then

$$
\phi(x)=\sum_{\alpha=1}^{\infty}\left[\int_{a}^{b} f(s) S(s) y_{\alpha}(s) d s\right] y_{\alpha}(x)
$$

converges uniformly on $X$ and $S(x)[f(x)-\phi(x)] \equiv 0$ on $X$.

For since

$$
\begin{aligned}
S(x) y_{i}(x) & =\lambda_{i} \int_{a}^{b} S(x) K(x ; s) y_{i}(s) d s \\
& =\lambda_{i} \int_{a}^{b} y_{i}(s) S(s) K(s ; x) d s \quad(i=1,2, \cdots),
\end{aligned}
$$

in view of $(\mathrm{H} 2)$, we have that

$$
\int_{a}^{b} f(s) S(s) y_{i}(s) d s=\left(1 / \lambda_{i}\right) \int_{a}^{b} g(s) S(s) y_{i}(s) d s .
$$

Hence

$$
\begin{aligned}
\phi(x) & =\sum_{\alpha=1}^{\infty}\left(1 / \lambda_{\alpha}\right) y_{\alpha}(x)\left[\int_{a}^{b} g(s) S(s) y_{\alpha}(s) d s\right] \\
& =\sum_{\alpha=1}^{\infty}\left[\int_{a}^{b} H(x ; s) S(s) y_{\alpha}(s) d s\right]\left[\int_{a}^{b} g(s) S(s) y_{\alpha}(s) d s\right]
\end{aligned}
$$

In view of the above Lemma 2 we then have that each component of $\phi(x)$ converges uniformly on $X$. We may then integrate $\int_{a}^{b} y_{i}(x) S(x)[f(x)-\phi(x)] d x$ term by term and obtain that this integral is zero for each $y_{i}(x)$. It then follows from Theorem 4 that $S(x)[f(x)-\phi(x)] \equiv 0$ on $X$.

CoRollary. If $|S(x)| \neq 0$ on $X$, then for every vector $f(x)$ of the form (22) the series $\phi(x)$ converges uniformly and represents $f(x)$ on $X$. 
THEOREM 7. If $f(x)$ is of the form (22) and $g(x)$ is of the form

$$
g(x)=\int_{a}^{b} K(x ; s) h(s) d s,
$$

where $h(x)$ is a vector each of whose components is continuous on $X$, then the series $\phi(x)$ converges uniformly on $X$ to the vector $f(x)$.

For by Theorem 6 we have that the series

$$
\sum_{\alpha=1}^{\infty} y_{\alpha}(x)\left[\int_{a}^{b} y_{\alpha}(s) S(s) g(s) d s\right]
$$

converges uniformly on $X$. From (24) it then follows that

$$
f(x)-\phi(x)=\int_{a}^{b} K(x ; s)\left[g(s)-\sum_{\alpha=1}^{\infty} y_{\alpha}(s) \int_{a}^{b} y_{\alpha}(t) S(t) g(t) d t\right] d s,
$$

and since $S(x)[f(x)-\phi(x)] \equiv 0$ on $X$, we have from (H3) that $\phi(x)=f(x)$ on $X$.

4. Remarks. If for a definitely self-adjoint vector integral equation the matrix $S(x)$ is the identity matrix $E$, then the matrix kernel of the vector integral equation is symmetric and the vector integral equation corresponds to a single integral equation with a closed symmetric kernel.

Bliss $\nmid$ has treated a system of ordinary linear differential equations of the first order which may be written in vector form as

$$
y^{\prime}=[A(x)+\lambda B(x)] y,
$$

where $A(x)$ and $B(x)$ are matrices of $n$ rows and columns whose elements are continuous on the interval $X$ and $y$ is a vector with $n$ components, each of which is continuous and has a continuous first derivative on $X$ satisfying (26). With (26) are associated boundary conditions

$$
M y(a)+N y(b)=0,
$$

where $M$ and $N$ are constant matrices such that the matrix $\|M, N\|$ is of rank $n$. According to Bliss the boundary value problem (26), (27) is said to be self-adjoint if the differential equations and also the boundary conditions of the adjoint system are equivalent to its own for all values of $\lambda$ by means of a transformation $z=T(x) y$, where the elements of $T(x)$ are real, single-valued, and have continuous first derivatives on $X$, and such that $|T(x)| \neq 0$ on $X$.

The boundary value problem (26), (27) is said to be definitely self-adjoint if the matrix $S(x) \equiv T^{*}(x) B(x)$ is symmetric, the bilinear form $\bar{f} S(x) f$ is non-

† Bliss, loc. cit. 
negative on $X$, and this form vanishes identically for a vector $f(x)$ which is a solution of an equation of the type

$$
f^{\prime}(x)=A(x) f(x)+B(x) g^{\prime} x
$$

where $g(x)$ is an arbitrarily chosen vector whose components are continuous, only when $f(x) \equiv 0$ on $X$.

We may assume without loss of generality that the system

$$
y^{\prime}=A(x) y,
$$

together with the boundary conditions (27), is incompatible. Then (26), (27) is equivalent to the vector integral equation

$$
y(x)=\lambda \int_{a}^{b} G(x ; s) B(s) y(s) d s
$$

where $G(x ; s)$ is the Green's matrix for (29), (27). The matrix kernel of (30) is of the form $H(x ; s) S(s)$, where $H(x ; s)=G(x ; s) T^{*-1}(s)$ and $S(x)=T^{*}(x)$ $B(x)$. By using the properties of the Green's matrix for a definitely selfadjoint boundary value problem as established by Bliss, $\uparrow$ it may be shown that (30) is a definitely self-adjoint vector integral equation, according to the definition of this paper. It is also to be noted that condition (H3) used in defining a definitely self-adjoint vector integral equation is somewhat weaker than the corresponding condition given by Bliss in defining a definitely self-adjoint boundary value problem.

If now the elements of $A(x)$ and $B(x)$ in (26) are not continuous, but merely Lebesgue summable, we define as a solution of (26) a vector $y$ whose components are absolutely continuous and which satisfies (26) "almost everywhere" on $X$. The system (26), (27) will then be said to be self-adjoint if the differential equations and also the boundary conditions of the adjoint system are equivalent to its own for all values of $\lambda$ by means of a transformation $z=T(x) y$, where now the elements of $T(x)$ are real, single-valued, such that $|T(x)| \neq 0$ on $X$, and merely absolutely continuous. By analogy with the definition of Bliss, the system (26), (27), when the elements of $A(x)$ and $B(x)$ are merely Lebesgue summable, is said to be definitely self-adjoint when $S(x)$ $\equiv T^{*}(x) B(x)$ is symmetric, $\bar{f} S(x) f \geqq 0$, and if $\bar{f}(x) S(x) f(x)=0$ "almost everywhere" on $X$ for a solution of (28), where $g(x)$ is an arbitrary summable vector, then $f(x) \equiv 0$. System (26), (27) is then equivalent to the vector integral equation (30), where now it is understood that the integral is taken in the sense of Lebesgue. If, however, the elements of $B(x)$ are continuous, while

† See Bliss, loc. cit., pp. 577-581. 
the elements of $A(x)$ are summable, then the matrix kernel of (30) satisfies the continuity condition of (H1), and (30) is again a definitely self-adjoint vector integral equation, according to the definition of this paper.

In defining a definitely self-adjoint vector integral equation we have imposed on the matrices $S(x)$ and $H(x ; s)$ the continuity condition of (H1). Clearly a related treatment for a vector integral equation in which the matrix kernel satisfies weaker continuity conditions may be carried out, just as the treatment of a single scalar integral equation has been carried through under weaker conditions.

University of Chicago,

Chicago, Ill. 